\title{
pMSSM studies with ATLAS and CMS
}

\author{
William James FAWCETT*, on behalf of the ATLAS and CMS Collaborations \\ University of Oxford (GB) \\ E-mail: william.fawcettecern.ch
}

The ATLAS and CMS collaborations conducted a large number of searches for supersymmetry during Run-1 of the LHC. No statistically significant excess was found and so limits have been placed on the masses of supersymmetric particles. These limits are usually interpreted in terms of low-scale simplified models, however, these models can fail to capture the complexity of full MSSM models and therefore provide over-optimistic exclusion. Both the ATLAS and CMS collaborations have embarked upon large-scale studies to combine their most sensitive searches for supersymmetry and analysed their impact on the 19-parameter phenomenological MSSM; allowing them to take into account all relevant dark matter, heavy flavour and precision electroweak measurements, as well as the constraints from the LHC searches. The results allow one to see where there is room for new physics still to be hiding, in some cases at surprisingly low masses.

Fourth Annual Large Hadron Collider Physics Conference

13-18 June 2016

Lund, Sweden

${ }^{*}$ Speaker. 


\section{Introduction}

The Standard Model (SM) of particle physics provides a successful framework to explain the phenomena observed in the strong, electromagnetic and weak interactions of fundamental particles. However, this framework cannot explain major astrophysical observations, such as the dark matter abundance in the Universe. Supersymmetry (SUSY) is a proposed extension to the SM that predicts a fermion partner to ever SM boson and vice versa. It can provide a wealth of solutions to the problems facing the SM, including the hierarchy problem related to the mass of the Higgs boson and also providing a credible dark matter candidate in the case of $R$-parity conservation.

As a result, searches for SUSY have been central to the LHC programme during Run-1 (and now continuing during Run-2). The ATLAS [1] and CMS [2] collaborations have conducted several searches at centre of mass energies of 7 and $8 \mathrm{TeV}$ and found no evidence for SUSY. This leaves the question, how do we interpret the results of these searches on SUSY? One approach is to use simplified models, which typically contain two or three supersymmetric particle mass parameters and fixed branching fractions between the decays, and provide a simple way to interpret the null results of searches. Search results interpreted in the simplified model context place limits in a 2D plane on the masses of the supersymmetric particles in question. Several examples of simplified model limits in the gluino-neutralino mass plane are shown in Figure 1, and shows that each model imposes a different limit on the gluino and neutralino masses. In the broader context of the full MSSM, it is less clear what supersymmetric particle masses have been excluded.

On the other hand, the full weak-scale $R$-parity conserving MSSM has around 120 parameters [4], and is therefore too complex to explore directly. An alternative is the 19-parameter phenomenological MSSM (pMSSM). Both the ATLAS and CMS collaborations have produced large-scale studies to asses the impact of a combination of searches in the context of the pMSSM, with ATLAS combining 22 searches [5], and CMS 11 [6].

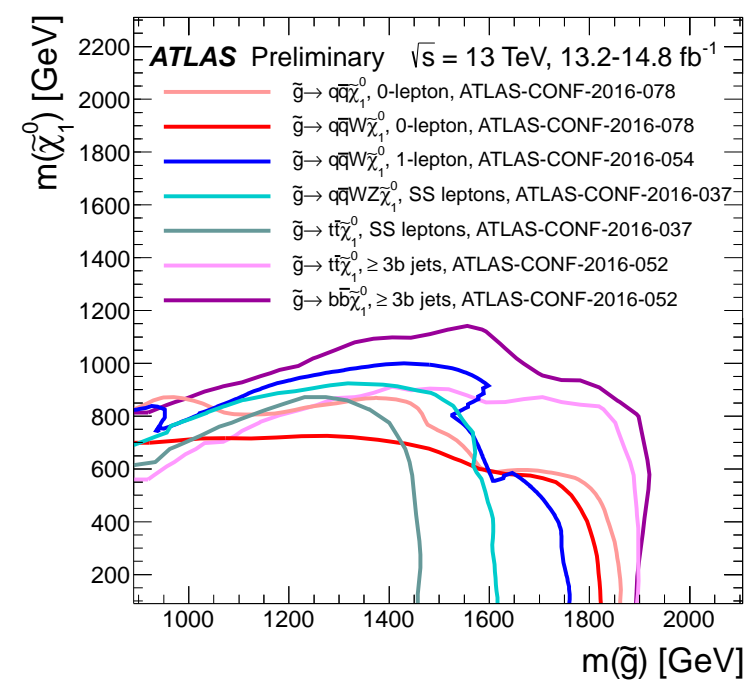

Figure 1: Simplified model exclusion limits at 95\% CL, in the gluino-neutralino mass plane from various Run-2 ALTAS searches. Figure taken from Ref. [3]. 


\section{The Phenomenological MSSM}

The majority of the 120 parameters of the $R$-parity conserving MSSM are associated with flavour changing neutral currents (FCNC) or CP-violating phases, both of which are tightly constrained by experiment. Therefore, by applying a series of experimentally motivated constraints, one can reduce the number of parameters to 19. The following constraints are applied by both the ATLAS and CMS collaborations:

1. No flavour changing neutral currents.

2. No additional $\mathrm{CP}$ violating phases beyond the CKM matrix.

3. Each $1^{\text {st }}$ generation sfermion is degenerate with it's $2^{\text {nd }}$ generation counterpart.

This parameter set (a full list of which can be found in the experimental papers [5, 6]) is small enough to explore with available computing resources, yet large enough to provide a good degree of flexibility. It allows for a correlations between different types of studies to be examined, such as dark matter searches and heavy-flavour measurements, as well as allowing the complete SUSY particle spectrum. Finally, it was required that the lightest neutralino, $\tilde{\chi}_{1}^{0}$ was the lightest SUSY particle (LSP).

\section{Model generation and classification}

Even with 19 parameters, the unbounded pMSSM is too large to scan exhaustively. Therefore, both collaborations restricted the range of each parameter to simultaneously provide a parameter space viable for the LHC and to make the scan computationally tractable Refs [5, 6]. Notably, the masses of each sparticle were constrained with an upper limit of 4 (3) TeV in the ATLAS (CMS) case, motivated to ensure a high density of models in reach of the LHC. Lower limits on sparticle masses were also applied to avoid constraints from the LEP experiments.

Points (or models) within the remaining subspace were then required to satisfy all relevant experimental measurements, including indirect constraints from precision electroweak measurements, muon $g_{\mu}-2$, the invisible width of the $Z$, Higgs mass and heavy flavour measurements such as $B R\left(B_{s} \rightarrow \mu \mu\right)$. ATLAS uniformly sampled $500 \times 10^{6}$ points in the subspace and then applied the above experimental constraints leaving $300 \times 10^{3}$ viable models, whereas CMS constructed a Bayesian prior and used Markov chain Monte Carlo techniques to find $20 \times 10^{6}$ viable models. In the ATLAS case, models were required to not over-saturate the dark matter relic density measured by the Planck Collaboration [9], however it was not assumed that the LSP was to be the sole constituent of dark matter, so only the upper limit was placed on the relic density. CMS applied no constraint on dark matter.

In order to quantify the impact of the searches, the models were classified as excluded or not excluded by each of the searches considered by the collaborations. ATLAS tested all $300 \times 10^{3}$ of their models against the 22 searches by first simulating each model at particle level ${ }^{1}$ (requiring the simulation of approximately 33 billion events) and classifying models as excluded, not excluded or

\footnotetext{
${ }^{1}$ Particle level: not taking into account detector effects.
} 
uncertain based on model independent limits. The uncertain models then went through fast detector simulation [7] and were tested with the original analysis codes. CMS simulated 7200 models, including the full detector response, and tested each with the 11 original analysis codes. ATLAS treated each model with the CLs prescription and classified the model as excluded if $\mathrm{CL}_{s}<0.05$ (i.e. excluded at $95 \% \mathrm{CL}_{s}$ ), CMS constructed likelihoods based on the results of the individual searches and performed a Bayesian analysis.

\section{Results and Discussion}

\section{1 supersymmetric particles}

It can be challenging to interpret the impact of searches on multi-dimensional parameter spaces such as the pMSSM. One of the most generic ways of presenting the impact of searches is by looking at the effect of the analyses on the overall production cross-section. This is shown for CMS in Figure 2. The most likely production cross-section is approximately $100 \mathrm{fb}$ before the CMS searches, however, after the searches are applied this is reduced by an order of magnitude, demonstrating the impact of direct searches for SUSY on the pMSSM.

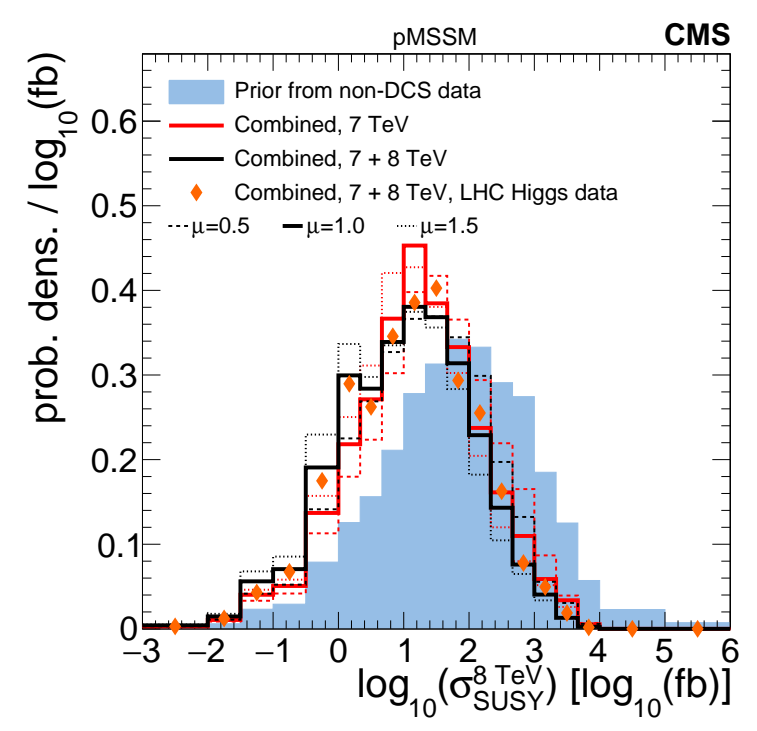

Figure 2: Impact of the CMS searches on the probability density of the logarithm of the cross-section for inclusive sparticle production in $8 \mathrm{TeV} p p$ collisions. The blue filled histogram shows the distribution of models before the CMS searches. The black and red histograms show the distribution after the 7, and $7+8 \mathrm{TeV}$ searches respectively, and the orange diamonds show the distribution after the $7+8 \mathrm{TeV}$ searches and Higgs boson measurements. Figure from Ref. [6].

Another method is to project the pMSSM onto two dimensional planes; the most relevant parameters typically being sparticle masses. Figure 3 shows the distribution of models before the experimental searches and also as the survival probability, or equivalently fraction of models excluded, after the searches for both ATLAS and CMS separately. Even though the two collaborations took different approaches to selecting, generating and excluding models, the results are broadly similar between the experiments, showing a good complementarity between the two. It 
appears as though the ATLAS model set has fewer models with $m\left(\tilde{\chi}_{1}^{0}\right) \lesssim 100 \mathrm{GeV}$ than the CMS model set, however, this is an artefact of the binning. There is, however, a discontinuity in the distribution of models around $m\left(\tilde{\chi}_{1}^{0}\right) \sim 100 \mathrm{GeV}$ most obvious in the ATLAS plots. There are fewer models in this region than in the others as only models with a bino-like LSP can satisfy the LEP constraint on the mass of the chargino; models with $m\left(\tilde{\chi}_{1}^{ \pm}\right) \lesssim 100 \mathrm{GeV}$ are excluded, affecting models with a wino- or Higgsino-like LSP for which the neutralino and chargino are degenerate. When comparing the exclusion power of the two experiments (as shown in Figure 3(b) and 3(d)) again there is a good correspondence and both show that all models with a gluino mass less than $500 \mathrm{GeV}$ were excluded by the 7 and $8 \mathrm{TeV}$ searches. Figure 3(b) also shows that the excluded region has a good congruence with the overlaid simplified model limit, which assumes the pair production of gluinos with decays to a pair of quarks and a neutralino, $\tilde{g} \rightarrow q q \tilde{\chi}_{1}^{0}$. This is perhaps surprising, given the increased complexity of the PMSSM, where all sparticle production and decay modes are available.

Figure 4 shows the probability density for the stop mass before and after the CMS searches, and also the fraction of models excluded by the ATLAS searches in the stop-neutralino mass plane. The CMS prior histogram (Figure 4(a)) shows that it is difficult to find models with light stops, $m\left(\tilde{t}_{1}\right) \lesssim 500 \mathrm{GeV}$, in the pMSSM, which is mainly due to the requirement of a Higgs boson with mass $\approx 125 \mathrm{GeV}$. This can also be seen from the ATLAS plot, Figure 4(b), as there are no models in the region $m\left(\tilde{\chi}_{1}^{0}\right) \lesssim 300 \mathrm{GeV}-m\left(\tilde{t}_{1}\right)$. Figure $4(\mathrm{~b})$ further shows that the exclusion of models is different from what is implied by the simplified model limit for models with light neutralinos, but is actually stronger for models with heavier neutralinos, and so the simplified model limit is not a good approximation in this case. Lastly, we see that models with $m\left(\tilde{t}_{1}\right) \sim 250 \mathrm{GeV}$ are still viable in the pMSSM, even after the combined Run-1 searches.

\subsection{Higgs to invisibles}

Given the parametric freedom available in the pMSSM, one can explore more than just the impact of searches on sparticle masses. Both the ATLAS and CMS collaborations have searched for Higgs bosons decaying invisibly, and set limits on the cross-section and branching ratio for this type of decay. If

$$
m\left(\tilde{\chi}_{1}^{0}\right) \leq \frac{1}{2} m(h)
$$

then the following decay can occur: $h \rightarrow \tilde{\chi}_{1}^{0} \tilde{\chi}_{1}^{0}$, which has the signature of an invisible Higgs decay. Therefore, the limits on this process can be used to constrain SUSY models. This is demonstrated by Figure 5(a) which shows that neutralinos with $m\left(\tilde{\chi}_{1}^{0}\right) \lesssim 65 \mathrm{GeV}$ are less-likely after taking into account the aforementioned limits. In principle, if no invisible Higgs decay is found, this might rule out models with neutralinos with masses given by Equation 4.1. However, Figure 5(b) shows that although only a small fraction of models are excluded by the limit on the branching ratio, the distribution of models rises with decreasing $\operatorname{BR}\left(h \rightarrow \tilde{\chi}_{1}^{0} \tilde{\chi}_{1}^{0}\right)$. Given that it is more likely to have a smaller value of $\operatorname{BR}\left(h \rightarrow \tilde{\chi}_{1}^{0} \tilde{\chi}_{1}^{0}\right)$, the experimental collaborations will have to work hard to discover neutralinos in this way.

\subsection{Dark Matter}

The major difference between the ATLAS and CMS approaches to the PMSSM was the con- 


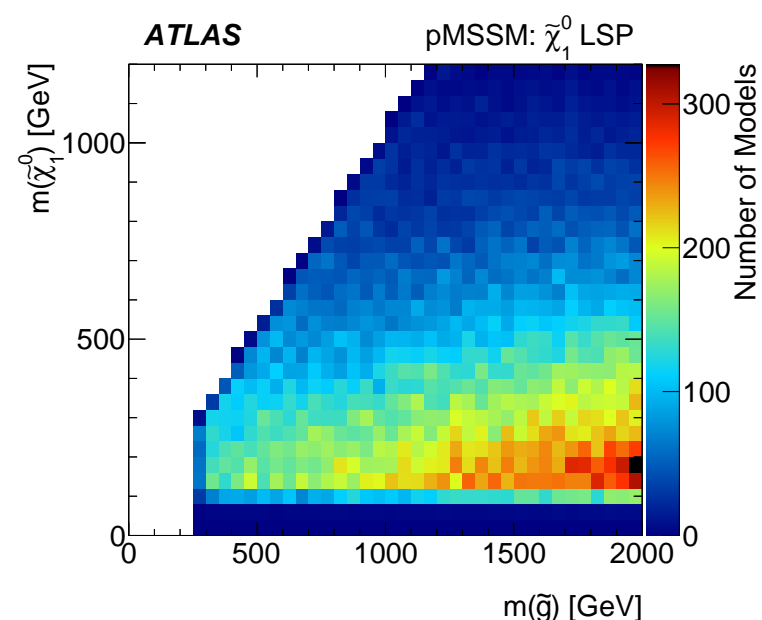

(a) ATLAS: before

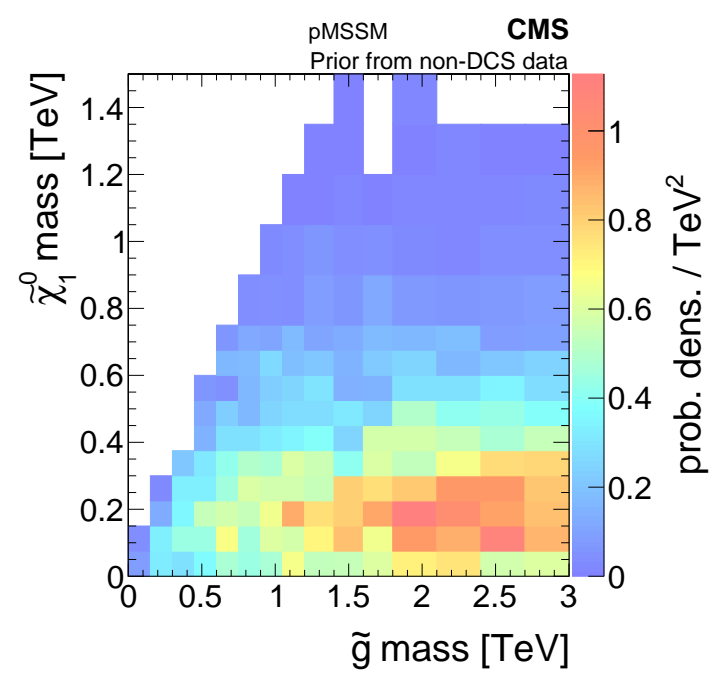

(c) CMS: before

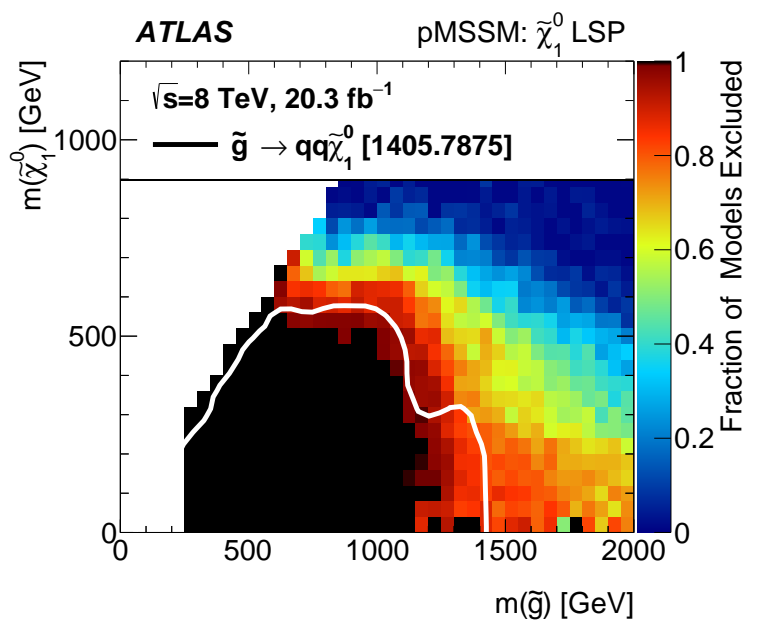

(b) ATLAS: after

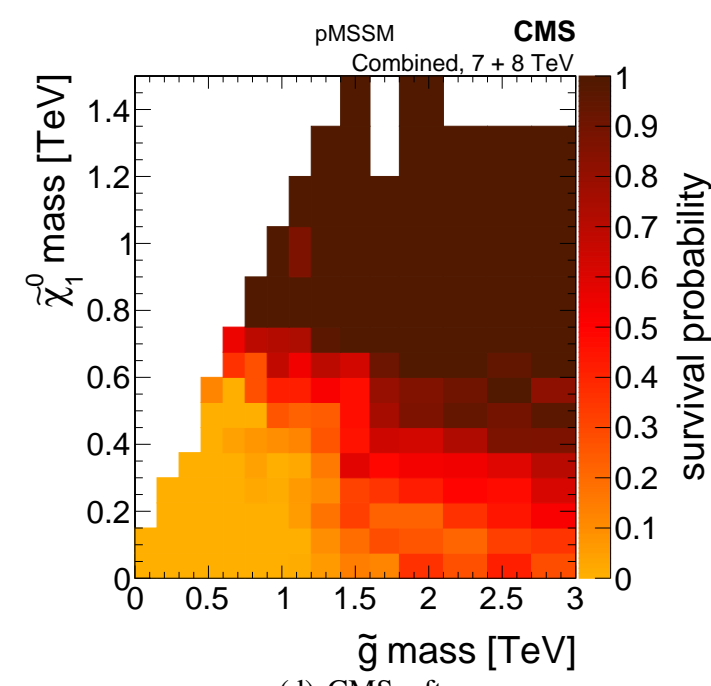

(d) CMS: after

Figure 3: The distribution of models before the combined searches (left) and the fraction of models excluded/survival probability after the combined searches (right) for the ATLAS (top) and CMS (bottom) studies, in the gluino-neutralino mass plane. White squares indicate regions where no models were generated. The ATLAS figures are from Ref. [5], the CMS figures are from Ref. [6]. The simplified model limit overlaid in (b) is taken from Ref. [8] and assumes pair production of gluinos, with each gluino directly decaying to two quarks and a neutralino $\tilde{g} \rightarrow q q \tilde{\chi}_{1}^{0}$

straint on dark matter. ATLAS required that the LSP did not over-saturate the dark matter relic density as measured by the Planck Collaboration, i.e. $\Omega_{\tilde{\chi}_{1}^{0}} h^{2}<0.1208$ [9], whereas CMS applied no such constraint. This resulted in a different phenomenology of the models considered by each collaboration. Figure 6(a) show that from the CMS scan, models are more likely to produce too much dark matter. Furthermore, after the $7+8 \mathrm{TeV}$ searches, the probability density shifts towards smaller valued of $\Omega_{\tilde{\chi}_{1}^{0}} h^{2}$, meaning that searches are more sensitive to models that produce more dark matter.

Imposing the dark matter constraint has several effects on the phenomenology of models. 


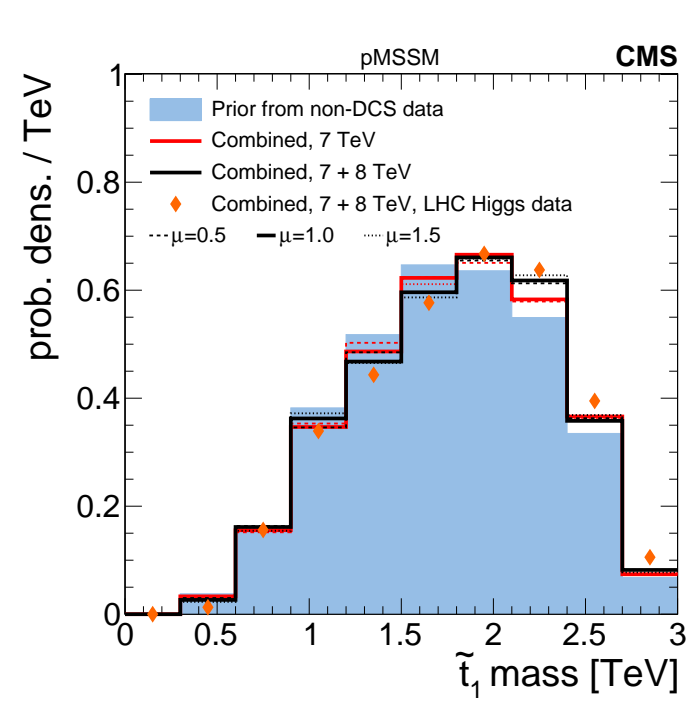

(a)

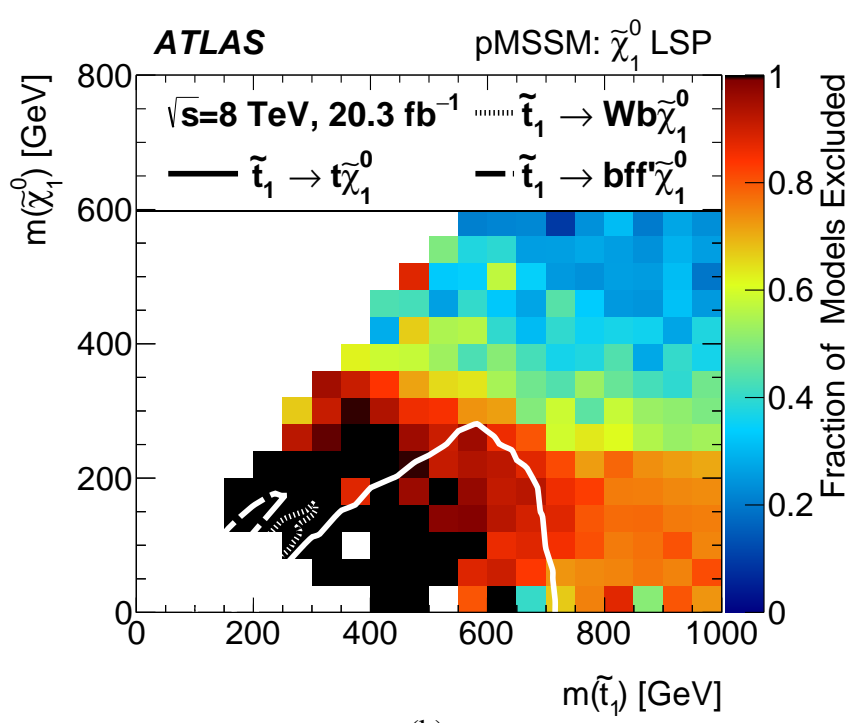

(b)

Figure 4: Left: histograms showing the probability density for the stop mass before and after the combined CMS searches (shading as in Figure 2), figure taken from Ref. [6]. Right: the fraction of models excluded in each bin by the combined ATLAS searches in the stop-neutralino plane, figure taken from Ref. [5].

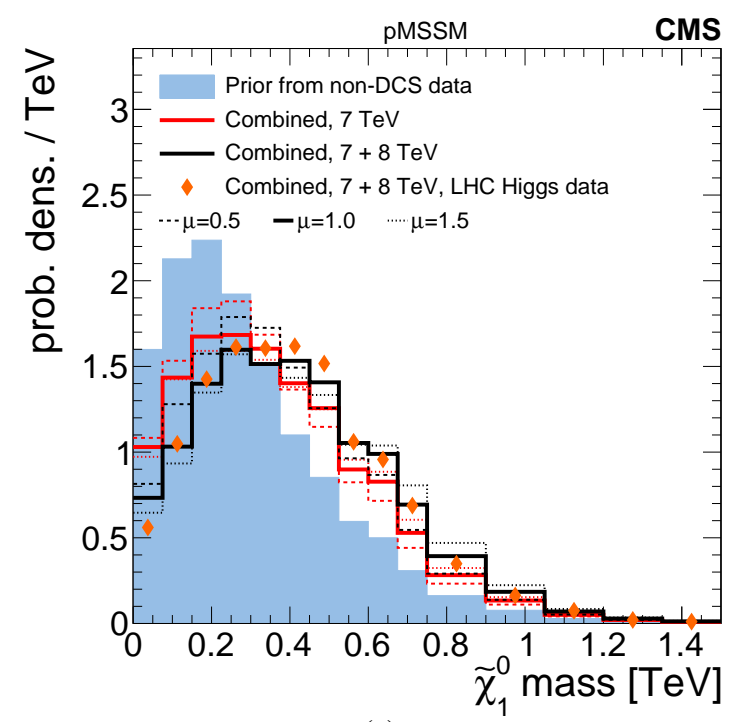

(a)

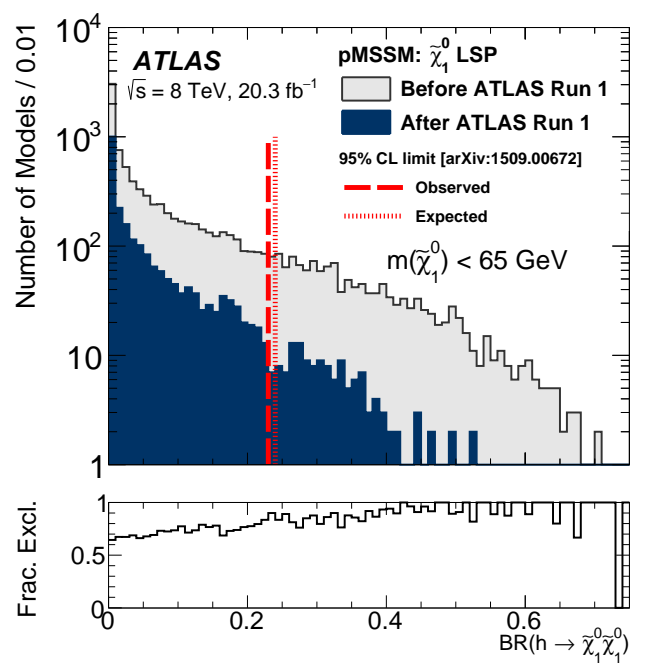

(b)

Figure 5: Left: the probability density of the neutralino mass before and after the CMS searches, taken from Ref. [6], shading as in Figure 2. Right: the distribution of the branching fraction of the invisible decay $h \rightarrow \tilde{\chi}_{1}^{0} \tilde{\chi}_{1}^{0}$, taken from Ref. [5], with the experimental limit overlaid with the red dashed lines. 
One result is that the LSP must have some mechanism to reduce the dark matter relic density. One such mechanism occurs if the LSP has at least one other SUSY particle sufficiently close in mass such that they can annihilate and reduce the dark matter relic density; such particles are referred to as co-annihilators. Models with a wino- or Higgsino-like LSP are accompanied by a degenerate chargino which acts as a co-annihilator, this effectively limits the maximum mass of the neutralino to approximately 1 (2.5) TeV for pure Higgsino (wino) neutralinos (see Ref. [10], for example). Models with a bino-dominated LSP do not necessarily have a degenerate chargino, and so some other annihilation mechanism is needed, for example by co-annihilation with another sparticle. As a result, it is more difficult to find models with a bino-dominated LSP in the pMSSM, as unless the LSP happens to have another sparticle close in mass, the model will produce too much dark matter. ATLAS had scan through a factor of 24 more points in order to find roughly the same number of viable bino-LSP models as wino- or Higgsino-LSP models. The results in an interesting set of models with bino-dominated LSPs, as shown in Figure 6(b), with several notable features. The stalactite-like structures in the region $m\left(\tilde{\chi}_{1}^{0}\right)<100 \mathrm{GeV}$ correspond to cases where the neutralino mass is half of either the $Z$ or $h$ bosons and can resonantly annihilate $\tilde{\chi}_{1}^{0} \tilde{\chi}_{1}^{0} \rightarrow Z / h$. Beyond $m\left(\tilde{\chi}_{1}^{0}\right)>100 \mathrm{GeV}$ there are several, overlapping, triangular structures correspond to other types of co-annihilation, for example $\tilde{\chi}_{1}^{0} \tilde{\tau} \rightarrow \gamma \tau$. The efficiency of the co-annihilation is related to the mass splitting, the coupling strength (the type of co-annihilator sparticle) and the mass of the neutralino [11], so the lower diagonal of the triangular structures are bounded by the maximally efficient annihilation mechanism. The vertical edges are bounded by constraints from previous experimental searches that were imposed during model generation.

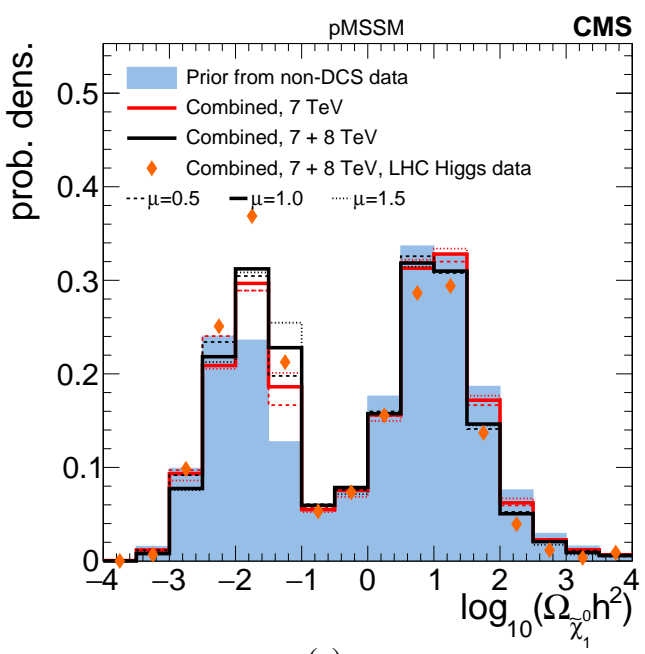

(a)

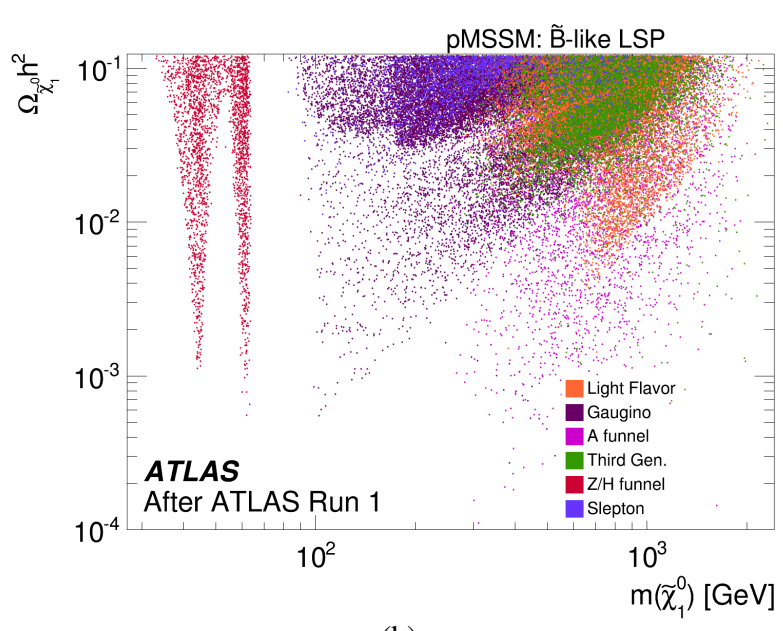

(b)

Figure 6: Left: probability density of the dark matter relic density before and after the CMS searches, shading as in Figure 2, taken from Ref. [6]. Right: Each point corresponds to a model that survived the ATLAS searches and has a bino-dominated LSP, colour coded by the co-annihilation mechanism, in the plane of the neutralino mass versus the dark matter relic density. Figure taken from Ref. [5]. 


\section{Summary}

The ATLAS and CMS collaborations have both performed many searches for SUSY with data from Run-1 of the LHC. The results of these searches are usually interpreted by setting limits on sparticle masses in terms of simplified models. However, in the broader context of the MSSM it is less clear what sparticle masses have been excluded. To address this problem, the ATLAS and CMS collaborations have combined 22 and 11 of their searches (respectively), and interpreted the impact of these results on the 19-parameter pMSSM. Although the two collaborations chose different approaches in selecting models in the pMSSM for their studies, the experimental results are broadly similar, showing a good complementarity between the two. Comparing simplified model limits to the exclusion in the pMSSM shows that the limits are not always a good approximation, and it can be an over-simplification leading to over-stringent limits on SUSY particles. This, however, is promising for naturalness arguments as models with lights stops $\left(m\left(\tilde{t}_{1}\right) \sim 250 \mathrm{GeV}\right)$ are still viable. Other searches and measurements, such as the search for an invisible decay of the Higgs boson can also aid in the search for SUSY. Furthermore, the parametric freedom of the pMSSM also allows interpretation of dark matter results, and the impact that constraints from non-LHC experiments such as the Planck satellite can have on SUSY.

Finally, Figure 7 shows the sensitivity of the combined ATLAS Run-1 searches on each sparticle available in the pMSSM. For full details on the ATLAS and CMS analyses, see Refs. [5] and [6] respectively. For further reading, ATLAS has also produced a similar study investigating the impact of electroweak searches on the pMSSM [12].

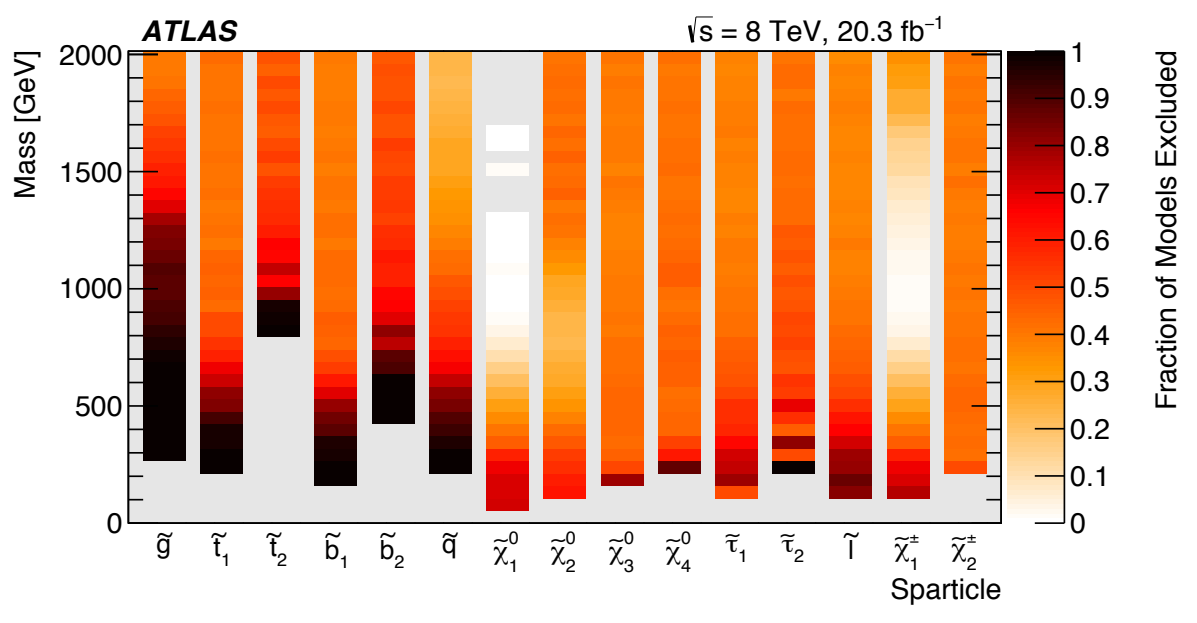

Figure 7: A summary of the sensitivity of ATLAS to different types of supersymmetric particles in the pMSSM. Each vertical bar is a 1D projection of the sparticle mass, with the colour coding representing the fraction of models excluded by the ATLAS searches in each bin. Figure taken from Ref. [3]. 


\section{Acknowledgements}

The author would like to thank Pembroke College, Oxford, for their generosity in supporting his attendance at the Fourth Annual Large Hadron Collider Physics conference.

\section{References}

[1] ATLAS Collaboration, The ATLAS Experiment at the CERN Large Hadron Collider, JINST 3 (2008) S08003.

[2] CMS Collaboration, The CMS experiment at the CERN LHC, JINST 3 (2008) S08004.

[3] https://twiki.cern.ch/twiki/bin/view/AtlasPublic/SupersymmetryPublicResults

[4] MSSM Working Group, The minimal sypersymmetric standard model: group summary report, in GDR (Groupement De Recherche) - Supersymétrie Montpellier, France, 1998 arXiv:hep-ph/9901246.

[5] ATLAS Collaboration, Summary of the ATLAS experiment's sensitivity to supersymmetry after LHC Run 1 - interpreted in the phenomenological MSSM, JHEP 10 (2015) 134.

[6] CMS Collaboration, Phenomenological MSSM interpretation of CMS searches in pp collisions at $\sqrt{s}=7$ and $8 \mathrm{TeV}$, [arXiv:1606.03577] submitted to JHEP.

[7] ATLAS Collaboration, The ATLAS Simulation Infrastructure, Eur. Phys. J. C 70 (2010) 823.

[8] ATLAS Collaboration, Search for squarks and gluinos with the ATLAS detector in final states with jets and missing transverse momentum using $\sqrt{s}=8 \mathrm{TeV}$ proton-proton collision data, JHEP 1409 (2014) 176.

[9] Planck Collaboration, Planck 2015 results. XIII. Cosmological parameters, [arxiv:1502.01589].

[10] N. Arkani-Hamed, A. Delgado and G.F. Giudice, The Well-Tempered Neutralino, Nucl. Phys. B741 (2006).

[11] J. Ellis, T. Falk, K.A. Olive, Neutralino-Stau Coannihilation and the Cosmological Upper Limit on the Mass of the Lightest Supersymmetric Particle, Phys. Lett. B. 444 (1998) 367.

[12] ATLAS Collaboration, Dark matter interpretations of ATLAS searches for the electroweak production of supersymmetric particles in $\sqrt{s}=8 \mathrm{TeV}$ proton-proton collisions, [arXiv:1608.00872], submitted to JHEP. 